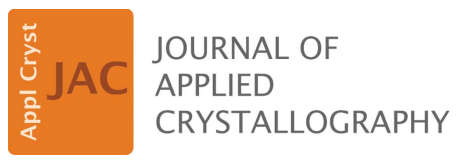

ISSN 1600-5767

Received 27 February 2018

Accepted 14 March 2018

Keywords: X-ray optics; quartz; Bragg reflection; Darwin bandwidth; X-ray analyzers.

Supporting information: this article has supporting information at journals.iucr.org/j
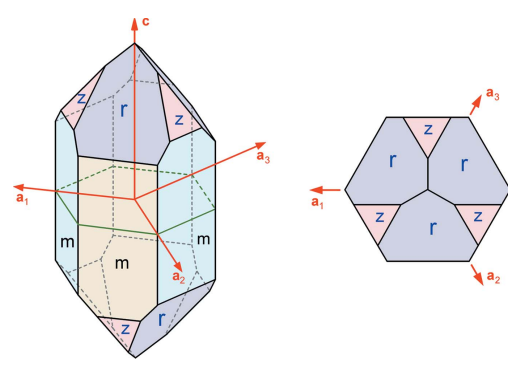

C 2018 International Union of Crystallography

\section{Correct interpretation of diffraction properties of quartz crystals for X-ray optics applications. Corrigendum}

\author{
Xian-Rong Huang, ${ }^{\mathrm{a} *}$ Thomas Gog, ${ }^{\mathrm{a}}$ Jungho Kim, ${ }^{\mathrm{a}}$ Elina Kasman, ${ }^{\mathrm{a}}$ Ayman H. Said, \\ Diego M. Casa, ${ }^{a}$ Michael Wieczorek, ${ }^{a}$ Marcelo G. Hönnicke ${ }^{b}$ and Lahsen Assoufid ${ }^{a}$
}

\footnotetext{
aAdvanced Photon Source, Argonne National Laboratory, 9700 South Cass Avenue, Argonne, Illinois 60439, USA, and bInstituto de Ciencias da Vida e da Natureza, Universidade Federal da Integracao Latino-Americana, 2044 Foz do Iguacu, Parana, 85867-970, Brazil. *Correspondence e-mail: xiahuang@aps.anl.gov
}

Errors about left-handed (laevorotatory) quartz and right-handed (dextrorotatory) quartz in the paper by Huang, Gog, Kim, Kasman, Said, Casa, Wieczorek, Hönnicke \& Assoufid [J. Appl. Cryst. (2016), 51, 140-147] are corrected.

In the paper by Huang et al. (2016), the authors erroneously stated that the helical arrangement of the Si atoms in quartz is the same as the 'optical handedness'. The authors are grateful to Professor A. M. Glazer at Physics Department, University of Oxford, for pointing out that this statement is incorrect (see also Glazer, 2018). In fact, the two types of handedness are opposite to each other (Glazer \& Stadnicka, 1986). Therefore, in the right-handed hexagonal coordinate system, the unit cell with the three Si atoms located at $(u, 0,0),(-u,-u, 1 / 3)$ and $(0, u, 2 / 3)$ and the six $\mathrm{O}$ atoms at $(x, y, z),(y, x, 2 / 3-z),(-y$, $x-y, 2 / 3+z),(-x, y-x, 1 / 3-z),(y-x,-x, 1 / 3+z)$ and $(x-y,-y,-z)$ corresponds to right-handed quartz (space group $\mathrm{P3}_{2} 21$ ) with right-handed optical rotation (dextrototation), although here the three $\mathrm{Si}$ atoms form a left-handed helix (or left-handed screw, LS). Accordingly, still in the righthanded hexagonal coordinate system, the unit cell with the $\mathrm{Si}$ atoms located at $(u, u,-1 / 3),(-u, 0,0)$ and $(0,-u, 1 / 3)$ (forming a right-handed screw, RS) and the $\mathrm{O}$ atoms at $(x, x-$ $y, z-1 / 3),(y, y-x, 1 / 3-z),(-y,-x, 1 / 3+z),(-x,-y,-z)$, $(y-x, y, z)$ and $(x-y, x, 2 / 3-z)$ corresponds to left-handed quartz (space group $P 3_{1} 21$ ) with left-handed optical rotation (laevorotation). Since the authors have adopted $u=0.4699, x=$ $0.4141, y=0.2681$ and $z=0.1188$, right-handed quartz $\left(P 3_{2} 21\right)$ corresponds to the $z(+)$ setting in the right-handed hexagonal coordinate system of Donnay \& Le Page (1978), and lefthanded quartz $\left(P 3_{1} 21\right)$ corresponds to the $z(-)$ setting in the right-handed hexagonal coordinate system. These settings correspond to the 'reverse settings' (widely adopted in the $\mathrm{X}$-ray community) so that reflection $10 \overline{11}$ is stronger than $10 \overline{1} 1$, and $30 \overline{31}$ is much stronger than $30 \overline{3} 1$.

In summary, except for the words 'right- or left-handed hexagonal coordinate systems' and 'left-handed helix', all the other words 'left-handed' should be 'right-handed' and the words 'right-handed' should be 'left-handed' in the original paper and the original supporting information. A revised version of the paper and the supporting information with all 
the corrections has been deposited as new supporting information to this paper.

\section{References}

Donnay, J. D. H. \& Le Page, Y. (1978). Acta Cryst. A34, 584-594.
Glazer, A. M. (2018). J. Appl. Cryst. 51, 915-918.

Glazer, A. M. \& Stadnicka, K. (1986). J. Appl. Cryst. 19, 108122.

Huang, X.-R., Gog, T., Kim, J., Kasman, E., Said, A. H., Casa, D. M., Wieczorek, M., Hönnicke, M. G. \& Assoufid, L. (2018). J. Appl. Cryst. 51, 140-147. 